\title{
Association of hand grip strength with disease activity, disability and quality of life in children and adolescents with Juvenile Idiopathic Arthritis
}

\author{
Ahmed Mohammed Rashed ${ }^{1 \dagger}$, Noha Abdel-Wahab ${ }^{1,2 \dagger}$, Ehab M. M. Moussa ${ }^{3}$ and Nevin Hammam ${ }^{1,4^{*}}$
}

\begin{abstract}
Background: Juvenile idiopathic arthritis (JIA) affects wrist and hand joints leading to decrease hand function and patients' daily living activities. The assessment of hand grip strength (HGS) in children and adolescents with JIA is of major importance, and the association of HGS with JIA disease activity, disability and quality of life has not been explored. The primary objective of this study was to evaluate hand grip strength (HGS) in children and adolescents with Juvenile Idiopathic Arthritis (JIA) compared to matched healthy peers. The secondary objective was to explore the relationship between HGS and JIA disease activity, disability, and quality of life.
\end{abstract}

Methods: This study involved 23 patients with JIA and 46 age and sex matched healthy controls. Hand held dynamometer was used to evaluate HGS for all study participants. Anthropometric parameters for all study participants were measured. Disease activity, physical function, and quality of life were assessed for the JIA group using juvenile arthritis disease activity score (JADAS-27), juvenile arthritis functionality scale (JAFS), and pediatric quality of life inventory (PedsQL) respectively. Laboratory marker of inflammation, erythrocyte sedimentation rate $(E S R)$, and plain radiography of hands were performed for all patients.

Results: Hand grip strength of children and adolescents with JIA was significantly weaker compared to matched controls $(p<0.001)$. Hand grip strength had a significant inverse correlation with JADAS-27 $(r=-0.467, p=0.025)$, JAFS $(r=-0.650, p=0.001)$ and a significant direct correlation with PedsQL $(r=0.438, p=0.036)$. In addition, HGS was negatively correlated with ESR and duration of morning stiffness $(r=-0.489, p=0.018$ and $r=-0.201, p=0.359$, respectively). HGS was detected as an independent predictor of disease activity, disability, and quality of life in JIA patients in multivariate linear regression.

Conclusions: Assessment of HGS could be a simple non-invasive tool for assessing disease activity, disability and quality of life in JIA patients in clinical practice.

Keywords: Activity, Disability, Dynamometer, Hand strength, Juvenile Idiopathic Arthritis

\footnotetext{
* Correspondence: hammam@ualberta.ca

${ }^{\dagger}$ Ahmed Mohammed Rashed and Noha Abdel-Wahab contributed equally to

this work.

${ }^{1}$ Rheumatology and Rehabilitation Department, Faculty of Medicine, Assiut

University, Assiut, Egypt

${ }^{4}$ Department of Physical Therapy, Faculty of Rehabilitation Medicine,

University of Alberta, Corbett Hall, 8205114 Street, Edmonton, AB T6G2G4,

Canada

Full list of author information is available at the end of the article
}

(c) The Author(s). 2018 Open Access This article is distributed under the terms of the Creative Commons Attribution 4.0 International License (http://creativecommons.org/licenses/by/4.0/), which permits unrestricted use, distribution, and reproduction in any medium, provided you give appropriate credit to the original author(s) and the source, provide a link to the Creative Commons license, and indicate if changes were made. The Creative Commons Public Domain Dedication waiver (http://creativecommons.org/publicdomain/zero/1.0/) applies to the data made available in this article, unless otherwise stated. 


\section{Background}

Juvenile Idiopathic Arthritis (JIA) is the most common cause of childhood chronic arthritis, with the worldwide prevalence reported as 7-401 cases per 100,000 children [1]. Diagnosis of JIA in children and adolescents under 16 years old is challenging as the diagnosis is made after exclusion of other causes of chronic arthritis, and the clinical course is heterogeneous with possible systemic manifestations [2, 3].

JIA most commonly affects the musculoskeletal system with bilateral hand and wrist joints being predominantly involved in approximately $80 \%$ of the patients with significant short and long term disability [2-4]. Recurrent inflammation, localized and generalized osteoporosis, joints damage and deformities, and disturbance of muscle function predispose to weakness of the hand grip strength (HGS) in those children $[4,5]$. Progressive loss of hand strength and function could ultimately lead to hand disability and adversely impacts the children daily living activities, school performance, and their overall quality of life [6].

Hand dynamometer was used as an accurate method for assessment of HGS in normal children [7, 8]. Among adults with rheumatoid arthritis (RA), studies suggested that HGS measured by dynamometer being a routine evaluation in these patients [9], and can be used as a measure for disease activity in patients with RA [10-12]. Evaluating HGS either subjectively or objectively in children with different rheumatic diseases including JIA has been previously studied $[6,13,14]$. However, no studies to date determined the association between HGS, disease activity, disability and quality of life in children and adolescents with JIA.

The primary objective of this study was to evaluate HGS in children and adolescents with JIA compared to age and gender matched healthy peers. The secondary objective was to explore the relationship between HGS and JIA disease activity, disability, and quality of life.

\section{Methods}

\section{Study design and study participants}

A cross-sectional observational study including children and adolescents with JIA and age and gender matched healthy peers was conducted. JIA patients were recruited from the Rheumatology department at our hospital during the period of January to June 2013. Inclusion criteria were children and adolescents aged 10-16-years old who have been diagnosed with JIA according to the International League of Associations for Rheumatology (ILAR) classification criteria [15]. The control participants were selected from the patients' relatives. Children younger than 10 years old were excluded as the explanation and performance of the procedure were difficult under this age. Children with previous upper limb fractures or surgeries were also excluded. The local medical ethical review committee at the University approved all of the study procedures. Written informed consent was obtained from all children's parents or their first degree relatives before participation in the study.

\section{Demographic and disease characteristics}

Demographic data from all study participants and clinical data of JIA patients were collected. Disease activity was measured using the juvenile arthritis disease activity score (JADAS-27) which is ranging from 0 to 57 and patients were classified according to the defined cut-off values to either low $(\leq 2.7)$, moderate $(2.8-5.9)$, or high $(\geq 6)$ disease activity [16]. The degree of physical disability and functional impairment was evaluated using the juvenile arthritis functionality scale (JAFS) with total score of 30 [17]. JIA patients' quality of life was assessed using the pediatric quality of life Inventory v.4 questionnaire (PedsQL), with score of 100 as the best quality or no difficulty during action [18].

\section{Anthropometric measurements}

All study participants underwent body weight and height measurements using electronic scale and stadio-meter platform respectively, and body mass index (BMI) was calculated.

\section{Laboratory and radiological data}

First hour ESR (erythrocyte sedimentation rate) was calculated and normalized according to the JADAS-27 formula [19]. Conventional radiographic scan of both hands for all patients was done and was evaluated by a rheumatologist and by the same radiologist.

\section{Hand grip strength measurements}

The participants' HGS was measured using hand held dynamometer (Camry Digital Hand Dynamometer Grip Strength, Model EH101-37, China) with digital display and a standard adjustable hand for ideal grasp. Instructions and demonstrations were given to the participants according to the standard recommendations [20]. The device is designed for auto capturing the maximum achieved grip strength and displaying it in kilograms. Children and adolescents were instructed to grip the device for each hand as hard as possible for 3 consecutive times [21]. The measurement was done by the same investigator for all participants who was blind to the clinical data of the patients. The mean value of the three all-out gripping trials was considered as HGS measure for each hand.

\section{Statistical analysis}

Data were summarized using descriptive statistics, with mean and standard deviation (SD) for normally 
distributed continuous variables, median inter quartile range (IQR) for non-normally distributed continuous variables, and frequencies with percentages for dichotomous variables. Differences between patients and controls were tested using independent samples t-test. Relationship between HGS and JADAS-27 score, JAFS score and PedsQL was tested by Pearson correlations. Separate linear regression analyses of the association between HGS as an independent variable, and JADAS-27, JFAS, and PedsQL as continuous dependent variables were performed, and the results were expressed as beta-coefficient $(\beta)$ and $95 \%$ confidence interval (CI). Analysis was performed using the Statistical Package for Social Science version 16 (SPSS Inc.; Chicago, IL, USA). $P$ value $\leq 0.05$ was considered to be statistically significant.

\section{Results}

\section{Participants characteristics}

The study included 23 JIA patients ( 9 boys and 14 girls) and 46 controls (19 boys and 27 girls), with the average age of 13.3 and 12.7 years for boys and girls respectively. No significant differences in the demographic and anthropometric characteristics of the study participants were observed. The clinical parameters of the JIA patients are presented in (Table 1). All JIA disease subtypes, apart from the psoriatic group, were more or less equally represented in our study population.

\section{Hand grip strength of study participants}

All study participants were able to perform the HGS test using the electronic hand dynamometer. There were significant differences in the mean HGS values between the participants $(p<0.001)$ (Table 2$)$. In respect to gender, the mean HGS for both hands in JIA boys was significantly higher than in JIA girls $(p=0.013)$ and also in the controls participants $(p=0.017)$ (Fig. 1). HGS increased with age in both genders in JIA and control groups.

\section{Relationships between HGS and disease activity and disability variables}

The correlations between HSG value and patients' and disease variables are described in (Table 3). When regression analyses were performed with HGS as a predictor and JADAS-27, JFAS, and PedsQL as an outcome, persistent significant associations were found (Table 4). This analysis reveals a negative weak association between HGS and JADAS-27 ( $\beta=-0.128, p=0.035)$ and negative strong association with JAFS ( $\beta=-0.657, P=0.001)$, and positive strong association with PedsQL $(\beta=1.837, P=0.036)$.

\section{Discussion}

The importance of HGS assessment derives from the fact that grip strength was previously suggested as an
Table 1 Clinical, laboratory, and radiological characteristics of Juvenile Idiopathic Arthritis children and adolescents

\begin{tabular}{|c|c|}
\hline Characteristics of JIA patients & Number (\%) or Mean \pm SD \\
\hline \multicolumn{2}{|l|}{ Disease subtypes: } \\
\hline Systemic onset & $5(21.7 \%)$ \\
\hline Oligo-arthritis & $4(17.4 \%)$ \\
\hline Poly-articular, positive RF & $4(17.4 \%)$ \\
\hline Poly-articular, negative RF & $5(21.7 \%)$ \\
\hline Enthesitis related arthropathy & $4(17.4 \%)$ \\
\hline Psoriatic arthritis & $1(4.3 \%)$ \\
\hline Disease duration (years) & $4.5 \pm 2.9$ \\
\hline Morning stiffness (minutes) & $30 \pm 29$ \\
\hline $\begin{array}{l}\text { Presence of local arthritis } \\
\text { (hand and/or wrist) }\end{array}$ & $12(52.2 \%)$ \\
\hline JADAS-27 total score, median (IQR) & $7.6(2-17)$ \\
\hline Low & $6(26.1 \%)$ \\
\hline Moderate & $1(4.3 \%)$ \\
\hline High & $16(69.6 \%)$ \\
\hline JAFS score, total score & $6.2 \pm 6.5$ \\
\hline Upper body segment & $1.1 \pm 1.7$ \\
\hline Hands score & $2.1 \pm 2.5$ \\
\hline Lower limbs & $3.1 \pm 3$ \\
\hline Total score after excluding both hands & $4.1 \pm 4.3$ \\
\hline PedsQL, total score & $64.8 \pm 27.2$ \\
\hline Physical health & $61.8 \pm 28.8$ \\
\hline Psycho-social health & $65.8 \pm 28.4$ \\
\hline \multicolumn{2}{|l|}{ Steroids use } \\
\hline Current Systemic steroids users & $15(65.2 \%)$ \\
\hline Dose (mg/day), median (IQR) & $20(10-20)$ \\
\hline Cumulative dose, mean $\pm S D$ & $481 \pm 473.8$ \\
\hline DMARDs users & $20(87 \%)$ \\
\hline ESR $(\mathrm{mm} / \mathrm{hr})$ & $34.3 \pm 22.2$ \\
\hline \multicolumn{2}{|l|}{ Radiological findings } \\
\hline Juxta-articular osteopenia & 19 (82.6\%) \\
\hline Soft tissue swelling & $11(47.8 \%)$ \\
\hline Narrow joint spaces & 7 (30.4\%) \\
\hline Deformities & $6(26.1 \%)$ \\
\hline Erosions & $5(21.7 \%)$ \\
\hline
\end{tabular}

$J A$ juvenile idiopathic arthritis, RF rheumatoid factor; min, minutes, JADAS-27 juvenile arthritis disease activity score, JAFS juvenile arthritis functionality scale, PedsQL pediatric quality of life, ESR Erythrocyte Sedimentation Rate, DMARDs disease modifying anti-rheumatic drugs

easy and inexpensive indicator of health status and an outcome measure for different diseases [22]. Moreover, in a recent large prospective study, HGS was assessed as risk of cardiovascular diseases and their mortality [23]. In our study, we observed a significant lower HGS of both hands in patients with JIA when compared with 
Table 2 Hand grip strength measurement in Juvenile Idiopathic Arthritis patients and controls

\begin{tabular}{llll}
\hline Hand grip strength & \multicolumn{2}{c}{ Mean \pm SD } & P-value \\
\cline { 2 - 3 } & Patients & Controls & \\
\hline Dominant hand & $13.7 \pm 6.6$ & $20.5 \pm 5.7$ & 0.001 \\
Non-dominant hand & $13.2 \pm 6.4$ & $20.1 \pm 6$ & $<0.001$ \\
Both hands & $13.4 \pm 6.5$ & $20.3 \pm 5.8$ & $<0.001$ \\
\hline
\end{tabular}

their matched healthy controls. A significant higher HGS of both hands in boys compared with girls was similarly observed in both patients and controls, and the difference significantly increased with age. The most important finding was the significant positive association observed between HGS and JIA disease activity, disability and negative association with quality of life among JIA. In radiographic findings, the only significant association we observed was the lower HGS in patients with narrow joint spaces.

Hand-held dynamometer had been used and validated in children and adolescents in previous national studies for determination of normative values [7, 8]. Although Jamar-like dynamometer is a standard grip strength measurement tool, it is a larger and heavier instrument and may therefore be more difficult for children to use [24]. The use of electronic hand dynamometer was more applicable for use in our patient population. Weak HGS in JIA patients was previously showed by Dunn who used a modified Sphygmomanometer to evaluate HGS in children aged 3-7 years with different rheumatic disorders including 13 patients with JIA compared to healthy children [13]. In 2003, Lindehammar described the changes in HGS that occur in patients with JIA over a 2 year follow-up [14]. Our observations regarding the
Table 3 Correlation coefficients between hand grip strength with patients and disease variables

\begin{tabular}{lll}
\hline Variables & HGS $(r$ value $)$ & $P$ value \\
\hline Age (years) & 0.461 & 0.001 \\
BMI of patients (kg/m2) & 0.257 & 0.237 \\
Disease duration & -0.201 & 0.359 \\
MS (minutes) & -0.559 & 0.038 \\
JADAS - 27 & -0.467 & 0.025 \\
Total JAFS & -0.650 & 0.001 \\
$\quad$ JAFS hand score & -0.635 & 0.001 \\
JAFS without hand score & -0.608 & 0.002 \\
Total PedsQL score & 0.438 & 0.036 \\
Physical health & 0.462 & 0.026 \\
Psycho-social health & 0.363 & 0.088 \\
Cumulative dose of steroids & -0.253 & 0.363 \\
DMARDs duration & -0.333 & 0.151 \\
ESR (mm/hr) & -0.489 & 0.018 \\
\hline
\end{tabular}

$B M I$ Body mass index, MS morning stiffness, JADAS-27 juvenile arthritis disease activity score, JAFS juvenile arthritis functionality scale, PedsQL pediatric quality of life, DMARDs disease modifying anti-rheumatic drugs, ESR erythrocyte sedimentation rate

changes in HGS according to the age and gender difference were consistent with the difference previously reported in patients with JIA [13], and in healthy children and adolescents [7, 25].

Although HGS has been previously assessed in JIA patients, no data regarding the relationship with JIA disease activity, and disability was reported. The present study revealed a negative correlation between HGS and disease activity which is in accordance with the previous studies in patients with adult onset RA $[10,12]$. HGS

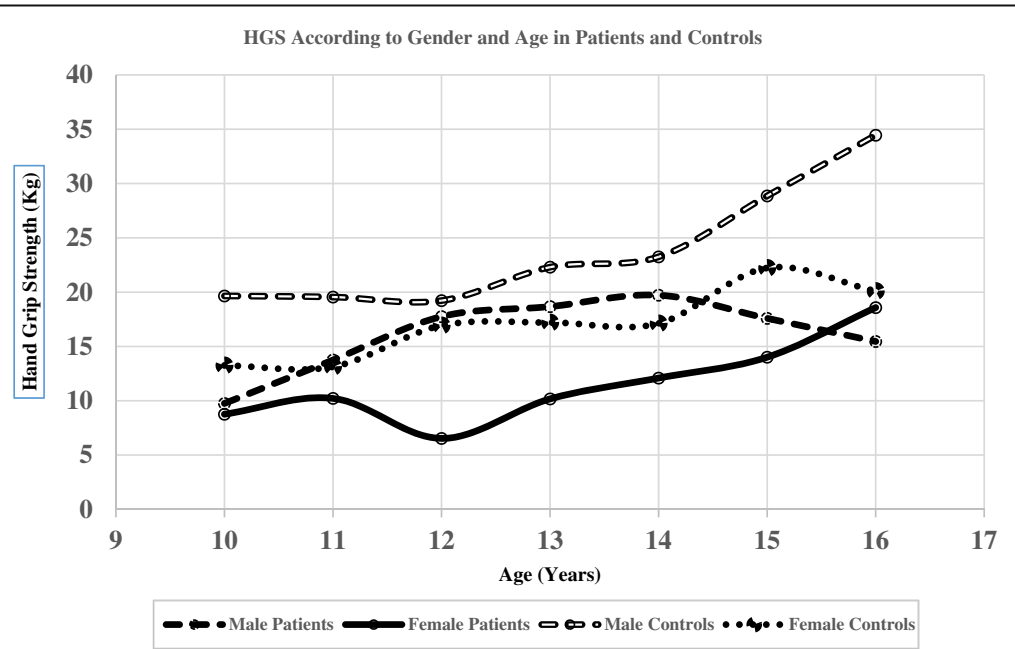

Fig. 1 Distribution of HGS according to genders and age in JIA and controls. Difference in the HGS for both gender in JIA and controls. The mean HGS in JIA boys was significantly higher than JIA girls and also the same in the controls participants. In addition, HGS significantly increased with age in both gender in JIA and control groups 
Table 4 Linear regression analysis for the effect of HGS on JADAS-27, JAFS, and PedQL outcome among JIA patients

\begin{tabular}{llllll}
\hline Variables & Beta & SE & Adjusted $R^{2}$ & $95 \%$ Cl & $P$ \\
\hline JADAS-27 & -0.128 & 0.053 & 0.181 & $(-0.238-0.018)$ & .025 \\
JAFS & -0.657 & 0.167 & 0.396 & $(-1.005-0.309)$ & .001 \\
PedsQL & 1.837 & 0.822 & 0.154 & $(0.128-3.546)$ & .036
\end{tabular}

JADAS-27 juvenile arthritis disease activity score, JAFS juvenile arthritis functionality scale, PedsQL pediatric quality of life, SE standard error, $\mathrm{Cl}$ confidence interval, $R^{2} \mathrm{R}$ square

measurement was recommended for self-assessment of RA disease activity in outpatient settings [12].

Hand and wrist joints are predominantly affected in patients with JIA $[6,26]$. Our results showed no statistically significant difference in HGS between patients with and without active arthritis of both hands and wrists. This observation was different from the results of Lindehammar who reported that children with active hand arthritis had less HGS, compared with patients without hand arthritis after 2 years of follow up [14]. The present study finding could be attributed to the fact that most of our patients experienced moderate to high disease activity which can lead to decreased physical activity in these children even in those without active hand arthritis. Also the chronic nature of the disease could be another explanation. However, none of these results could be confirmed because of the small sample size of patients evaluated.

One goal in the management of patients with JIA is to improve the patients' function and to prevent disability. HGS has been recognized as a valid technique to predict risk of active daily living disability [27]. The current results demonstrated an inverse significant correlation between HGS and physical function impairment assessed by total JAFS score and JAFS hands component score, similar to the results reported by Lindehammar using a subjective questionnaire [14]. The correlation remains persistently negative even after excluding the hand component from the total JAFS score, suggesting that HGS could reflect the overall functional impairment and movement restriction result from the disease itself rather than an indicator of local hand involvement. This result was in contrary to a previous study conducted in children with JIA to measure the limitation of physical activity in relation to HGS [6]. In Alaniz et al. [28] study, they used a self-assessment questionnaire and observed a significant correlation between wrist-related symptoms and problems at school. However our results were in agreement with similar studies in adult patients with RA [29] and in children with different disability [28].

JIA significantly impact the patients' QOL with marked reduction in all components; physical, social, emotional and school function. The present study results showed positive relation between HGS and the PedsQL total score. Our findings were in line with a previous study conducted in adult patients with RA whose HGS was negatively correlated with the health assessment questionnaire [29].

The study findings suggest that HGS could predict disease activity, functional impairment, and quality of life in JIA children and adolescents. The effect of a unit increase in HGS can predict the reduction in JADAS-27 and JAFS and increase PedsQL scores by $0.128,0.657$ and 1.837 respectively. The highest effect of HGS was detected on JAFS, with almost $40 \%$ of total JAFS can be explained by HGS, followed by JADAS-27 18.1\%, and PedsQL $15.4 \%$.

The relation between HGS and the radiographic changes in patients with JIA has not been previously evaluated, however, the study findings were in agreement with the results reported in patients with RA who showed an inverse correlation with the modified sharp score [29].

To the best of the authors' knowledge, this is the first study to examine the association between HGS and the disease activity, disability, and quality of life in JIA. The study findings highlight the feasibility of measuring HGS in children and adolescents with JIA in the clinical practice. Some limitations must be mentioned including the small sample size which make it difficult to evaluate the correlation between HGS and JIA disease onset subtypes, and also the cross sectional design of the study. Further longitudinal studies with larger sample size are needed to identify the determinants of HGS and to test whether improvement in strength reduces disease activity, disability and improve patients' quality of life.

\section{Conclusion}

In conclusion, the use of electronic hand dynamometer for assessment of HGS is a simple, non-invasive and inexpensive procedure which if applied in the outpatient settings could help the physician to evaluate the current disease activity, functional disability, and the quality of life and to better monitor the disease outcome in children and adolescents with JIA.

\section{Authors' contributions \\ AMR: Study idea, data collection and analysis and write first draft of the manuscript. NA-W: Study idea, data collection and analysis and revise the manuscript. EMMM: Data collection, revision of the manuscript. $\mathrm{NH}$ : Study idea, data collection, analysis and revise the manuscript, and submission. All authors read and approved the final manuscript.}

\section{Ethics approval and consent to participate}

The local medical ethical review committee at the University approved all of the study procedures. Written informed consent was obtained from all children's parents or their first degree relatives before participation in the study.

\section{Competing interests}

The authors declare that they have no competing interests. 


\section{Publisher's Note}

Springer Nature remains neutral with regard to jurisdictional claims in published maps and institutional affiliations.

\section{Author details}

${ }^{1}$ Rheumatology and Rehabilitation Department, Faculty of Medicine, Assiut University, Assiut, Egypt. ${ }^{2}$ Section of Rheumatology and Clinical Immunology, Department of General Internal Medicine, The University of Texas MD Anderson Cancer Center, Houston, Texas, USA. ${ }^{3}$ Department of Radiology, Assiut University Hospitals, Assiut, Egypt. ${ }^{4}$ Department of Physical Therapy, Faculty of Rehabilitation Medicine, University of Alberta, Corbett Hall, 8205 114 Street, Edmonton, AB T6G2G4, Canada.

Received: 30 April 2018 Accepted: 1 June 2018

Published online: 28 June 2018

\section{References}

1. Manners PJ, Bower C. Worldwide prevalence of juvenile arthritis why does it vary so much? J Rheumatol. 2002;29(7):1520-30.

2. Ravelli A, Martini A. Juvenile idiopathic arthritis. Lancet. 2007;369(9563):767-78.

3. Moorthy LN, Peterson MGE, Harrison MJ, Onel KB, Lehman TJA. Physical function assessment tools in pediatric rheumatology. Pediatr Rheumatol. 2008;6(1):1.

4. Lindehammar $\mathrm{H}$, Backman E. Muscle function in juvenile chronic arthritis. J Rheumatol. 1995;22(6):1159-65.

5. Hashkes PJ, Laxer RM. Medical treatment of juvenile idiopathic arthritis. JAMA. 2005;294(13):1671-84

6. Hoeksma AF, van Rossum MA, Zinger WG, Dolman KM, Dekker J, Roorda LD. High prevalence of hand- and wrist-related symptoms, impairments, activity limitations and participation restrictions in children with juvenile idiopathic arthritis. J Rehabil Med. 2014;46(10):991-6.

7. Ebrahim AHM. Normal handgrip strength for Egyptian children in Prepubertal developmental stage. Bull Fac Phys Ther. 2008;13(1):177-85.

8. Ervin RB, Fryar CD, Wang CY, Miller IM, Ogden CL. Strength and body weight in US children and adolescents. Pediatrics. 2014;134(3):782.

9. Shiratori AP, lop Rda R, Borges Junior NG, Domenech SC, Gevaerd Mda S. Evaluation protocols of hand grip strength in individuals with rheumatoid arthritis: a systematic review. Rev Bras Reumatol. 2014;54(2):140-7.

10. Sheehy C, Gaffney K, Mukhtyar C. Standardized grip strength as an outcome measure in early rheumatoid arthritis. Scand J Rheumatol. 2013;42(4):289-93.

11. Bircan C, Gunduz NE, Tekgul A, Cetin P, Onen F, Kizil R, et al. Grip ability test in rheumatoid arthritis patients: relationship with disease activity and hand-specific self-report questionnaires. Turkish J Rheumatol. 2014;29(3):160-7.

12. Espinoza F, Le Blay P, Coulon D, Lieu S, Munro J, Jorgensen C, et al. Handgrip strength measured by a dynamometer connected to a smartphone: a new applied health technology solution for the selfassessment of rheumatoid arthritis disease activity. Rheumatology (Oxford) 2016;55(5):897-901.

13. Dunn W. Grip strength of children aged 3 to 7 years using a modified sphygmomanometer: comparison of typical children and children with rheumatic disorders. Am J Occup Ther. 1993:47(5):421-8.

14. Lindehammar $\mathrm{H}$. Hand strength in juvenile chronic arthritis: a two-year follow-up. Acta Paediatr. 2003:92(11):1291-6.

15. Petty RE, Southwood TR, Manners P, Baum J, Glass DN, Goldenberg J, et al. International league of associations for rheumatology classification of juvenile idiopathic arthritis: second revision, Edmonton, 2001. J Rheumatol. 2004:31(2):390-2.

16. Bulatovic Calasan M, de Vries LD, Vastert SJ, Heijstek MW, Wulffraat NM. Interpretation of the juvenile arthritis disease activity score: responsiveness, clinically important differences and levels of disease activity in prospective cohorts of patients with juvenile idiopathic arthritis. Rheumatology (Oxford). 2014;53(2):307-12.

17. Filocamo G, Sztajnbok F, Cespedes-Cruz A, Magni-Manzoni S, Pistorio A, Viola $S$, et al. Development and validation of a new short and simple measure of physical function for juvenile idiopathic arthritis. Arthritis Rheum 2007:57(6):913-20

18. Varni JW, Seid M, Kurtin PS. PedsQL 4.0: reliability and validity of the pediatric quality of life inventory version 4.0 generic core scales in healthy and patient populations. Med Care. 2001;39(8):800-12.
19. Bazso A, Consolaro A, Ruperto N, Pistorio A, Viola S, Magni-Manzoni S, et al. Development and testing of reduced joint counts in juvenile idiopathic arthritis. J Rheumatol. 2009;36(1):183-90.

20. American Society of Hand Therapists. Clinical assessment recommendations. Chicago: The Society; 1992

21. Wang CY, Olson SL, Protas EJ. Test-retest strength reliability: hand-held dynamometry in community-dwelling elderly fallers. Arch Phys Med Rehabil. 2002;83(6):811-5.

22. Gubelmann C, Vollenweider P, Marques-Vidal P. Association of grip strength with cardiovascular risk markers. Eur J Prev Cardiol. 2016; https://doi.org/10. $1177 / 2047487316680695$

23. Leong DP, Teo KK, Rangarajan S, Lopez-Jaramillo P, Avezum A Jr, Orlandin A, et al. Prognostic value of grip strength: findings from the Prospective Urban Rural Epidemiology (PURE) study. Lancet (London, England). 2015; 386(9990):266-73.

24. Molenaar HM, Zuidam JM, Selles RW, Stam HJ, Hovius SE. Age-specific reliability of two grip-strength dynamometers when used by children. J Bone Joint Surg Am. 2008;90(5):1053-9.

25. Montalcini T, Ferro Y, Salvati MA, Romeo S, Miniero R, Pujia A. Gender difference in handgrip strength of Italian children aged 9 to 10 years. Ital J Pediatr. 2016;42:16.

26. Ravelli A, Martini A. Early predictors of outcome in juvenile idiopathic arthritis. Clin Exp Rheumatol. 2003:21(5 Suppl 31):89.

27. Al Snih S, Markides KS, Ottenbacher KJ, Raji MA. Hand grip strength and incident ADL disability in elderly Mexican Americans over a seven-year period. Aging Clin Exp Res. 2004;16(6):481-6.

28. Alaniz ML, Galit E, Necesito Cl, Hand Strength RER. Handwriting, and functional skills in children with autism. Am J Occup Ther. 2015;69(4):9.

29. Dedeoglu M, Gafuroglu U, Yilmaz O, Bodur H. The relationship between hand grip and pinch strengths and disease activity, articular damage, pain, and disability in patients with rheumatoid arthritis: romatoid artritli hastalarda elle kavrama ve tutma guclerinin hastalik aktivitesi, eklem hasari, Agri ve engellilik ile lliskisi. Turkish J Rheumatol. 2013;28(2):69-78.

\section{Ready to submit your research? Choose BMC and benefit from:}

- fast, convenient online submission

- thorough peer review by experienced researchers in your field

- rapid publication on acceptance

- support for research data, including large and complex data types

- gold Open Access which fosters wider collaboration and increased citations

- maximum visibility for your research: over $100 \mathrm{M}$ website views per year

At BMC, research is always in progress.

Learn more biomedcentral.com/submissions 